\title{
Critères de Complétude des Vecteurs Propres Généralisés d'une Classe d'Opérateurs Non Auto-adjoints Compacts ou à Résolvante Compacte et Applications
}

\author{
par \\ Marie-Thérèse AIMAR*, Abdelkader InTISSAR** et Jean-Martin PAOLI**
}

\begin{abstract}
In this work we establish sufficient conditions on the denseness of the generalized eigenvectors for a class of compact (or compact resolvent) non self adjoint operators. We can apply our results to operators arising in many fields, particularly in field's theory and in abstract second order differential equations. Our results generalize some results of Aimar and al [1] and [2], Macaev and Keldysh [11] and Dunford-Schwartz [9].
\end{abstract}

\section{§1. Position du Problème et Résultats}

Soit $E$ un espace de Hilbert sur $\mathbb{C}$ muni du produit scalaire $\langle$,$\rangle et de$ la norme associée \|\| et $K$ un opérateur compact.

Définition 1.1. On rappelle qu'un opérateur compact $K$ appartient á la classe $\ell_{p}$ de Carleman si la série $\sum_{n=1}^{\infty}\left[s_{n}(K)\right]^{p}$ converge oú $s_{n}(K), n=1,2, \ldots$ désigne la suite des valeurs propres de l'opérateur compact hermitien positif $\sqrt{K^{*} K}$. On dira qu'un opérateur compact $K$ appartient à la classe $\ell_{p}^{(0)}$ de Carleman généralisée si $s_{n}(K)=0\left(n^{-1 / p}\right)$ quand $n$ tend vers l'infini.

Ce travail a pour but de généraliser le résultat suivant:

Communiqué par T. Kawai, le 28 Mars, 1994. Revu le 10 Août, 1995.

1991 Mathematics Subject Classifications: 47A75, 35P10, 47B10, 47A10, 47F05

* Centre de Mathématiques et Informatique de l'Université de Provence. 39, Rue Joliot Curie, F-13453 Marseille cedex 13, France.

** Equipe d'analyse spectrale URA-CNRS n 2053.

Département de Mathématiques, Université de Corté, Quartier Grossetti, F-20250 Corte, France. 
Théorème 1.2 (Dunford-Schwartz [9], p: 1115). Soit $E$ un espace de Hilbert et $K$ un opérateur compact de classe $\ell_{p}$ de Carleman. On suppose que le plan complexe est divisé par $m$ demi-droites $\gamma_{1}, \gamma_{2}, \ldots, \gamma_{m}$ d'origine 0 telles que:

1) L'angle formé par deux demi-droites adjacentes soit strictement inférieur $\grave{a} \frac{\pi}{p}$.

2) Sur chacune de ces demi-droites, on ait: il existe un entier $N$ tel que $\left\|(\lambda I-K)^{-1}\right\|=O\left(|\lambda|^{-N}\right)$ quand $|\lambda|$ tend vers 0 .

Alors $K^{N}(E)$ est inclus dans l'adhérence de $\mathscr{E}$ où $\mathscr{E}$ est l'espace engendré par les vecteurs propres généralisés associés aux valeurs propres non nulles de $K$.

Remarque 1.3. La généralisation du théorème 1 se fait sur deux points :

a) On impose $s_{n}(K)=0\left(n^{-1 / p}\right)$ lorsque $n$ tend vers l'infini au lieu de $K$ appartient à $\ell_{p}$ ce qui représente une hypothèse moins restrictive sur l'opérateur $K$.

b) On autorise un angle égal à $\frac{\pi}{p}$.

Théorème 1.4. Soit $E$ un espace de Hilbert et $K$ un opérateur compact de classe $\ell_{p}^{(0)}$ de Carleman généralisée. On suppose que le plan complexe est divisé par $m$ demi-droites $\gamma_{1}, \gamma_{2}, \ldots, \gamma_{m}$ d'origine 0 telles que:

1) L'angle formé par deux demi-droites adjacentes soit inférieur ou égal à $\frac{\pi}{p}$.

il existe un entier $N$ tel que $\left\|(\lambda I-K)^{-1}\right\|=O\left(|\lambda|^{-N}\right)$ quand $|\lambda|$ tend vers 0. Alors $K^{N}(E)$ est inclus dans l'adhérence de $\mathscr{E}$ où $\mathscr{E}$ est l'espace engendré par les vecteurs propres généralisés associés aux valeurs propres non nulles de $K$.

Les outils essentiels à cette étude sont les suivants:

a) La majoration exponentielle de la résolvante pour des opérateurs appartenant à une classe de Carleman généralisée obtenue en utilisant une généralisation de l'inégalité de Carleman donnée par un théorème de Macaev [11].

b) Une version fine du principe de Phragmen-Lindelöf qui généralise le principe du maximum à des domaines non compacts du plan complexe.

Remarque 1.5. Si $K$ vérifie les hypothèses du théorème 1.4 alors il en est de même pour son adjoint $K^{*}$.

La démonstration du théorème 1.4 repose sur la proposition suivante:

Proposition 1.6. 1) Soit $\rho\left(K^{*}\right)$ l'ensemble résolvant de $K^{*}$ et $\varphi \in \mathscr{E}^{T}$, on considère la fonction $f: \rho\left(K^{*}\right) \rightarrow E$

$$
\lambda \longrightarrow f(\lambda)=\left(\lambda I-K^{*}\right)^{-1} \varphi
$$

Alors $f$ se prolonge en une fonction holomorhe de $\mathbb{C}-\{O\}$ dans $E$. 
2) Soit $\mathscr{E}^{T}$ l'orthogonal de $\mathscr{E}$ alors $\mathscr{E}^{T}$ ets stable par $K^{*}$.

3) Soit $K_{\mid \mathscr{E}^{T}}^{*}$ la restriction de $K^{*} \grave{a} \mathscr{E}^{T}$, alors $K_{\mid \mathscr{E}^{T}}^{*}$ est quasi-nilpotent, c'est-ádire que son spectre $\sigma\left(K_{\mid E^{T}}^{*}\right)$ est réduit à $\{O\}$.

4) $\mathrm{Si} K \in \ell_{p}^{(0)}$ alors $K_{\mid \mathscr{E}^{T}}^{*} \in \ell_{p}^{(0)}$.

5) Soit $\rho\left(K^{*}\right)$ l'ensemble résolvant de $K^{*}, \varphi \in \mathscr{E}^{T}$ et $\psi \in E$. On considère la fonction: $f: \rho\left(K^{*}\right) \rightarrow \mathbb{C}$

$$
\lambda \longrightarrow f(\lambda)=\left\langle\lambda^{N}\left(\lambda I-K^{*}\right)^{-1} \varphi, \psi\right\rangle
$$

Alors $f$ se prolonge en une fonction entière sur tout $\mathbb{C}$.

Démonstration. 1) Pour démontrer (1), on utilise le développement de Laurent de la résolvante de l'opérateur $K^{*}$ au voisinage de ses valeurs propres non nulles qui sont des points singuliers isolés. Soit $\lambda_{0} \in \sigma\left(K^{*}\right), \lambda_{0} \neq 0$ d'indice $m\left(\lambda_{0}\right)$. Il existe $\varepsilon>0$ tel que pour $0<\left|\lambda-\lambda_{0}\right| \leq \varepsilon$ on ait:

$$
\left(\lambda I-K^{*}\right)^{-1} \varphi=\sum_{n=0}^{\infty}\left(\lambda-\lambda_{0}\right)^{n} A_{n} \varphi+\sum_{n=1}^{m\left(\lambda_{0}\right)}\left(\lambda-\lambda_{0}\right)^{-n} A_{-n} \varphi
$$

où

- $A_{n}=\frac{1}{2 i \pi} \int_{C} \frac{\left(\lambda I-K^{*}\right)^{-1}}{\left(\lambda-\lambda_{0}\right)^{n+1}} d \lambda, C$ désignant le cercle de centre $\lambda_{0}$ ne contenant aucune autre valeur spectrale.

- $A_{-1}$ est la projection sur $\operatorname{Ker}\left(\lambda_{0} I-K^{*}\right)^{m\left(\lambda_{0}\right)}$ suivant $\operatorname{Im}\left(\lambda_{0} I-K^{*}\right)^{m\left(\lambda_{0}\right)}$

- $A_{n-1}=\left(K^{*}-\lambda_{0} I\right)^{-n} A_{-1}$ pour tout $n \leq-1$.

Comme $\operatorname{Ker}\left(\bar{\lambda}_{0} I-K\right)^{m\left(\lambda_{0}\right)}$ est inclus dans $\mathscr{E}$, on a les relations d'inclusion: $\mathscr{E}^{T} \subset\left[\operatorname{Ker}\left(\bar{\lambda}_{0} I-K\right)^{m\left(\lambda_{0}\right)}\right]^{T}=\operatorname{Im}\left(\lambda_{0} I-K^{*}\right)^{m\left(\lambda_{0}\right)}=\operatorname{Ker}\left(A_{-1}\right) \subset \operatorname{Ker}\left(A_{n}\right)$ pour tout $n \leq-1$. Par conséquent, pour tout $\varphi$ appartenant à $\mathscr{E}^{T}$, la partie singulière du développement en série de Laurent de $\left(\lambda I-K^{*}\right)^{-1} \varphi$ au voisinage d'une valeur propre non nulle s'annule. Il en résulte que $\left(\lambda I-K^{*}\right)^{-1} \varphi$ se prolonge analytiquement à $\mathbb{C}-\{O\}$.

2) Comme $\mathscr{E}$ est stable par $K$ alors $\mathscr{E}^{T}$ est stable par $K^{*}$.

3) Soit $K_{\mid \mathscr{E}^{T}}^{*}$ la restriction de $K^{*}$ à l'espace de Hilbert $\mathscr{E}^{T}$.

Supposons qu'il existe $\lambda_{1}$ non nul appartenant au spectre de $K_{\mid E^{T}}^{*}$; comme ce dernier est compact en tant que restriction d'un opérateur compact alors $\lambda_{1}$ est une valeur propre. Il existe donc un vecteur $\psi_{1}$ non nul appartenant à $\mathscr{E}^{T}$ tel que $K_{\mid \mathscr{E}^{T}}^{*} \psi_{1}=K^{*} \psi_{1}=\lambda_{1} \psi_{1}$. Il en résulte que $\psi_{1}$ appartient à $\operatorname{Ker}\left(\lambda_{1} I-K^{*}\right)=\left[\operatorname{Im}\left(\bar{\lambda}_{1} I-K\right)\right]^{T}$ et $\bar{\lambda}_{1}$ est une valeur propre de $K$. Soit $m\left(\bar{\lambda}_{1}\right)$ l'indice de $\bar{\lambda}_{1} ; \operatorname{Im}\left(\bar{\lambda}_{1} I-K\right)^{m\left(\bar{\lambda}_{1}\right)} \subset \operatorname{Im}\left(\bar{\lambda}_{1} I-K\right)$ et par conséquent $\psi_{1}$ appartient à $\left[\operatorname{Im}\left(\bar{\lambda}_{1} I-K\right)^{m\left(\bar{\lambda}_{1}\right)}\right]^{T}$. D'autre part $\psi_{1}$ appartient à $\mathscr{E}^{T}$ donc $\psi_{1}$ appartient à $\left.\left[\operatorname{Ker} \bar{\lambda}_{1} I-K\right)^{m\left(\bar{\lambda}_{1}\right)}\right]^{T}$.

Comme $E=\operatorname{Ker}\left(\lambda_{1} I-K^{*}\right)^{m\left(\lambda_{1}\right)} \oplus\left[\operatorname{Im}\left(\lambda_{1} I-K^{*}\right)^{m\left(\lambda_{1}\right)}\right]^{T}$, on en déduit $\psi_{1}=0$ d'où une contradiction. 
4) Il suffit de remarquer que les valeurs singulières $s_{n}\left(K_{\mid F}^{*}\right)$ sont inférieures ou égales à $s_{n}(K)$ pour tout sous espace de Hilbert $F$ de $E$.

5) Soit $\rho\left(K^{*}\right)$ l'ensemble résolvant de $K^{*}, \varphi \in \mathscr{E}^{T}$ et $\psi \in E$. On considère la fonction $f: \rho\left(K^{*}\right) \rightarrow \mathbb{C}$

$$
\lambda \longrightarrow f(\lambda)=\left\langle\lambda^{N}\left(\lambda I-K^{*}\right)^{-1} \varphi, \psi\right\rangle
$$

De (1) on déduit que $f$ se prolonge en une fonction holomorphe sur $\mathbb{C}-\{\boldsymbol{O}\}$ (on continue à noter ce prolongement $f$ ). De l'inégalité de Cauchy-Schwarz et de l'hypothèse (2) du théorème 1.4 on déduit que $f(\lambda)$ est bornée au voisinage de l'origine sur les demi-droites. Pour montrer que $f$ se prolonge en une fonction entière il suffit de montrer qu'elle est bornée au voisinage de l'origine. Pour cela on utilise une généralisation de l'inégalité de Carleman donnée par le théorème de Macaev [11] rappelé ci-dessous:

Théorème 1.7 (Macaev [11]). Soit $M(r)=\operatorname{Sup}_{|z|=r}\left\|(I-z K)^{-1}\right\|$ où $K$ est un opérateur quasi-nilpotent alors si $K \in \ell_{p}^{(0)}$ on a $\log M(r)=O\left(|z|^{p}\right)$ lorsque $|z| \rightarrow \infty$.

On applique ce théorème à l'opérateur $K_{\left.\right|^{T} T}^{*}$ qui en vérifie bien les hypothèses et on obtient $\log \left\|\left(I-\frac{1}{\lambda} K_{\mid \mathscr{E}^{T}}^{*}\right)^{-1}\right\|=O\left(|\lambda|^{-p}\right)$ quand $|\lambda| \rightarrow 0$. Par conséquent, $\forall \varepsilon>0$, il existe $C>0$ tel que pour tout $\lambda$ dans l'ensemble résolvant de $K_{\mid \mathscr{E}^{T} T}^{*}$ on ait:

$$
\left\|\left(I-\frac{1}{\lambda} K_{\mid \mathscr{E}^{T}}^{*}\right)^{-1}\right\| \leq C e^{\varepsilon|\lambda|^{-p}} \text {. On en déduit que: }
$$

$\forall \varepsilon>0$, il existe $C_{1}>0$ tel que pour tout $\lambda \neq 0$ on ait:

$$
|\lambda|^{N}\left\|\left(\lambda I-K_{\mid \mathscr{E}^{T} T}^{*}\right)^{-1}\right\| \leq \frac{|\lambda|^{N}}{|\lambda|}\left\|\left(I-\frac{1}{\lambda} K_{\mid \mathscr{E}^{T} T}^{*}\right)^{-1}\right\| \leq C_{1} e^{\varepsilon|\lambda|^{-p}}
$$

Il en résulte que $\forall \varepsilon>0$, il existe $c>0$ tel que pour tout $\lambda \neq 0$ on ait:

$$
|f(\lambda)| \leq c e^{\varepsilon|\lambda|^{-p}} \forall \psi \in E .
$$

Grâce à cette majoration, et du fait que $f$ est bornée sur les demi-droites, on peut appliquer le principe de Phragmen-Lindelöf et en déduire que $f$ est bornée au voisinage de l'origine et donc prolongeable en une fonction entière, ce qui achève la démonstration de la proposition.

Démonstration $d u$ théorème 1.4. Soit $\lambda$ appartenant à l'ensemble résolvant de $K^{*}$ alors on a $\lambda K^{* N-1}\left(\lambda I-K^{*}\right)^{-1}-I=K^{* N}\left(\lambda I-K^{*}\right)^{-1}$. Par récurrence sur $N$ on obtient: 


$$
K^{* N}\left(\lambda I-K^{*}\right)^{-1}=\lambda^{N}\left(\lambda I-K^{*}\right)^{-1}-\lambda^{N-1} I-\lambda^{N-2} K^{*}-\cdots \cdots-K^{* N-1}
$$

Soit $\varphi \in \mathscr{E}^{T}$ et $\psi \in E$ on a :

$$
\begin{aligned}
& \left\langle K^{* N}\left(\lambda I-K^{*}\right)^{-1} \varphi, \psi\right\rangle=\left\langle\lambda^{N}\left(\lambda I-K^{*}\right)^{-1} \varphi, \psi\right\rangle-\left\langle\lambda^{N-1} \varphi, \psi\right\rangle-\left\langle\lambda^{N-2} K^{*} \varphi, \psi\right\rangle \\
& -\cdots \cdots-\left\langle K^{* N-1} \varphi, \psi\right\rangle .
\end{aligned}
$$

D'après la proposition précédente, la fonction $\lambda \rightarrow\left\langle K^{* N}\left(\lambda I-K^{*}\right)^{-1} \varphi, \psi\right\rangle$ se prolonge en une fonction entière puisque c'est le cas pour $\left\langle\lambda^{N}\left(\lambda I-K^{*}\right)^{-1} \varphi, \psi\right\rangle$.

- D'autre part $\left|\left\langle K^{* N}\left(\lambda I-K^{*}\right)^{-1} \varphi, \psi\right\rangle\right| \leq\left\|K^{* N}\right\|\left\|\left(\lambda I-K^{*}\right)^{-1}\right\|\|\varphi\|\|\psi\|$. Comme $K^{*}$ est borné, $\operatorname{Lim}\left\|\left(\lambda I-K^{*}\right)^{-1}\right\|=0$ lorsque $|\lambda| \rightarrow \infty$. On en déduit que :

$\operatorname{Lim}\left\langle K^{* N}\left(\lambda I-K^{*}\right)^{-1} \varphi, \psi\right\rangle=0$ lorsque $|\lambda| \rightarrow \infty$ et donc que $\left\langle K^{* N}\left(\lambda I-K^{*}\right)^{-1} \varphi, \psi\right\rangle=0$ car c'est une fonction entière et bornée, donc constante d'après le théorème de Liouville; elle est d'autre part identiquement nulle car sa limite à l'infini est nulle. On en déduit que $K^{* N}\left(\lambda I-K^{*}\right)^{-1} \varphi=0$ $\forall \varphi \in \mathscr{E}^{T}$ et donc $K^{* N} \varphi=0$. Par conséquent $\left\langle K^{* N} \varphi, \psi\right\rangle=0 \quad \forall \psi \in E$ d'où $\varphi \in K^{N}(E)^{T}$ ce qui prouve que $\mathscr{E}^{T} \subset K^{N}(E)^{T}$ et par conséquent que $K^{N}(E) \subset \overline{\mathscr{E}}$.

De façon similaire, on obtient:

Théorème 1.8. Soit $E$ un espace de Hilbert et $K$ un opérateur compact de classe $\ell_{p}^{(0)}$ de Carleman généralisée. On suppose que le plan complexe est divisé par $m$ demi-droites $\gamma_{1}, \gamma_{2}, \ldots, \gamma_{m}$ d'origine 0 telles que:

1) L'angle formé par deux demi-droites adjacentes soit inférieur ou égal $\grave{a} \frac{\pi}{p}$.

2) Sur chacune de ces demi-droites, on ait $\left\|(\lambda I-K)^{-1}\right\|=O\left(\frac{1}{|\lambda|}\right) \begin{gathered}p \\ \text { quand } \lambda\end{gathered}$ tend vers zéro.

Alors les vecteurs propres généralisés de l'opérateur $K$ forment un système total dans E.

Démonstration. Il suffit de combiner le théorème précédent et le lemme suivant :

Lemme 1.9 ([9] p: 1042). Soit $E$ un espace de Hilbert et $K$ un opérateur borné sur $E$ alors on a l'alternative suivante:

Soit $E=\operatorname{Ker}(K)+\overline{\operatorname{Im}(K)}$, soit $\operatorname{Lim}\left\|(I-z K)^{-1}\right\|=\infty$ lorsque $|z|$ tend vers l'infini; $\operatorname{Ker}(K)$ et $\overline{\operatorname{Im}(K)}$ désignent respectivement le noyau de $K$ et l'adhérence de son image.

Une version du résultat précédent pour les opérateurs à résolvante compacte est donnée par:

Théorème 1.10. Soit $E$ un espace de Hilbert et $T$ un opérateur fermé de domaine dense dans $E$. On suppose que: 
1) $\exists p>0$ et $\exists \xi_{0} \in \rho(T)$ tel que $\left(\xi_{0} I-T\right)^{-1} \in \ell_{p}^{(0)}$

2) Il existe $m$ demi-droites $\gamma_{1}, \gamma_{2}, \ldots, \gamma_{m}$ d'origine 0 divisant le plan complexe telles que l'angle formé par deux demi-droites adjacentes soit inférieur ou égal $\grave{a} \frac{\pi}{p}$ et que sur chacune d'elles, on ait $\left\|(\lambda I-T)^{-1}\right\|=O\left(|\lambda|^{N}\right)$ quand $|\lambda|$ tend vers l'infini ( $N$ entier naturel)

Alors les vecteurs propres généralisés de l'opérateur $T$ forment un système total dans $E$.

Démonstration. On se ramène au cas où 0 appartient à l'ensemble résolvant. En posant $\mu=\frac{1}{\lambda}$ et $K=T^{-1}$, on a $(\mu I-K)^{-1}=\lambda I-\lambda(\lambda I-T)^{-1}$ $=\frac{1}{\mu}-\frac{1}{\mu^{2}}\left(\frac{1}{\mu} I-T\right)^{-1}$. Soit $\gamma_{1}, \gamma_{2}, \ldots, \gamma_{m}$ les demi-droites sur lesquelles $\left\|(\lambda I-T)^{-1}\right\|=O\left(|\lambda|^{N}\right)$ lorsque $|\lambda|$ tend vers l'infini. Sur les demi-droites images de $\gamma_{1}, \gamma_{2}, \ldots, \gamma_{m}$ par $z \rightarrow \frac{1}{z}$ on a:

$\left\|\left(\frac{1}{\mu} I-T\right)^{-1}\right\|=O\left(\frac{1}{|\mu|^{N}}\right)$ et $\left\|\frac{1}{\mu^{2}}\left(\frac{1}{\mu} I-T\right)^{-1}\right\|=O\left(\frac{1}{|\mu|^{N}}\right)$ dont on déduit que $\left\|(\mu I-K)^{-1}\right\|=O\left(\frac{1}{|\mu|^{N+2}}\right)$ quand $|\mu| \rightarrow 0$. D'après le théorème 1.4 , les vecteurs propres généralisés associés aux valeurs propres non nulles engendrent un sous espace $\mathscr{E}$ tel que $K^{N+2}(E)$ soit inclus dans l'adhérence de $\mathscr{E}$. Mais comme $K(E)$ est égal au domaine $D(T)$ de l'opérateur $T$ qui est dense dans $E$ il en résulte que l'adhérence de $K(E)$ est égale à $E$ et par récurrence on obtient:

$$
E \subset K\left(\overline{K^{N-1}(E)}\right) \subset \overline{K^{N}(E)} \text { donc } \overline{\mathscr{E}}=E=\overline{K^{N+2}(E)}
$$

Remarque 1.11. Le résultat de Lang-Locker [18] est un cas particulier de celui de Dunford-Schwartz ([9] corollaire 31, p: 1115) et donc du théorème 1.10 .

\section{§2. Quelques Applications}

\section{-Opérateur de Gribov:}

La théorie des champs de reggeons [10] est caractérisée par l'opérateur de Gribov $H_{\lambda}^{\prime}$ agissant sur l'espace de Bargmann [8]:

$$
E=\left\{\varphi: \mathbb{C}^{n} \longrightarrow \mathbb{C} \text { analytiques } ; \int_{\mathbb{C}^{n}} e^{-|z|^{2}}|\varphi(z)|^{2} d z d \bar{z}<\infty \text { et } \varphi(0)=0\right\}
$$

L'opérateur non auto-adjoint $H_{\lambda}^{\prime}$ est défini par: 


$$
\begin{aligned}
H_{\lambda}^{\prime}= & \lambda^{\prime} \sum_{j=1}^{n} A_{j}^{* 2} A_{j}^{2}+\mu \sum_{j=1}^{n} A_{j}^{*} A_{j}+i \lambda \sum_{j=1}^{n} A_{j}^{*}\left(A_{j}+A_{j}^{*}\right) A_{j} \\
& +\alpha \sum_{j=1}^{n-1}\left(A_{j+1} A_{j}^{*}+A_{j+1}^{*} A_{j}\right)
\end{aligned}
$$

où $A_{j}^{*}$ et $A_{j}$ sont respectivement les opérateurs de création et d'annihilation, $\left(\lambda^{\prime}, \mu, \lambda, \alpha\right)$ sont des paramètres réels et $i^{2}=-1$.

Une étude spectrale complète de $H_{\lambda}^{\prime}$ est donnée dans [12], [13] et [14]. Pour $\lambda^{\prime} \neq 0$, la densité du système des vecteurs propres généralisés de $H_{\lambda}^{\prime}$ est donnée dans [1], [2] ou [19].

Théorème 2.1 (Aimar-Intissar-Paoli [2]). 1) Pour $\lambda^{\prime} \neq 0, H_{\lambda}^{\prime}$ est un opérateur à résolvante de classe $\ell_{p}\left(p>\frac{n}{2}\right)$.

2) Pour $\lambda^{\prime} \neq 0$, il existe $\beta_{0}$ tel que $H_{\lambda}^{\prime}+\beta_{0} I$ soit accrétif et d'image numérique incluse dans un secteur d'ouverture $\frac{\pi}{p}$.

3) Pour $\lambda^{\prime} \neq 0$, le sous espace engendré par les vecteurs propres généralisés de $H_{\lambda}^{\prime}$ est dense dans l'espace de Bargmann.

Remarque 2.2. Pour $\lambda^{\prime}=0$, la question de la densité du système des vecteurs propres généralisés de $H_{\lambda}^{\prime}$ dans l'espace de Bargmann est encore ouverte. On donne néanmoins dans [3] et [4] quelques propriétés de régularité de cet opérateur limite.

\section{-Opérateurs différentiels à deux points dèterminés par $-D^{\mathbf{2}}$ :}

Dans [17] Lang et Locker ont donné une étude spectrale complète de l'opérateur $L=-D^{2}$ agissant sur $L_{2}[0,1]$ de domaine $D(L)=\left\{u \in H^{2}[0,1]\right.$; $B_{1}(u)=0$ et $\left.B_{2}(u)=0\right\}$ où :

$-H^{2}[0,1]$ est l'espace de Sobolev formé des fonctions $u \in C^{1}[0,1]$ avec $u^{\prime}$ absolument continue sur $[0,1]$ et $u^{\prime \prime} \in L_{2}[0,1]$

$-B_{1}$ et $B_{2}$ sont linéairement indépendants et donnés par:

$$
\begin{aligned}
& B_{1}(u)=a_{1} u^{\prime}(0)+b_{1} u^{\prime}(1)+a_{0} u(0)+b_{0} u(1) \\
& B_{2}(u)=c_{1} u^{\prime}(0)+d_{1} u^{\prime}(1)+c_{0} u(0)+d_{0} u(1)
\end{aligned}
$$

Certains de leurs résultats sont résumés dans le théorème suivant:

Théorème 2.3 (Lang-Locker [17]). 1) Si $a_{1} d_{1}-b_{1} c_{1}=0, a_{0} d_{0}-b_{0} c_{0}$ $=0$ et $a_{1} d_{0}-b_{0} c_{1}=a_{0} d_{1}-b_{1} c_{0}$, alors le spectre de L est soit vide soit égal à tout le plan complexe $\mathbb{C}$.

2) Si le spectre de L n'est ni vide ni tout le plan complexe $\mathbb{C}$, alors:

i) La résolvante de l'opérateur L est de Hilbert-Schmidt

ii) Le plan complexe est divisé par cinq demi droites issues de l'origine telles 
que l'angle formé par deux demi-droites adjacentes soit strictement inferieur à $\frac{\pi}{2}$ et que sur chacune on ait $\left\|(\lambda I-L)^{-1}\right\|=O\left(\frac{1}{\sqrt{|\lambda|}}\right)$ quand $|\lambda|$ tend vers l'infini

iii) Le système des vecteurs propres généralisés de Lest dense dans $L_{2}[0,1]$.

Remarque 2.4. Si le spectre de $L$ n'est ni vide ni tout le plan complexe $\mathbb{C}$ alors l'opérateur $L$ vérifie les hypothèses de notre thèorème 1.10 .

-Un problème aux limites pour une équation différentielle abstraite du second ordre:

Soit l'opérateur $L_{S} u(x)=-u^{\prime \prime}(x) ; 0 \leq x \leq 1$ associé aux conditions irrégulières de Stone $B_{1}(u)=u^{\prime}(0)+u^{\prime}(1)+u(1)=0$ et $B_{2}(u)=u(0)+u(1)=0$, qui sont un cas particulier de celles étudiées ci-dessus par Lang et Locker. On considère un espace de Hilbert $E$ et un opérateur linéaire $A$, de domaine $D(A)$ dense dans $E$ et fortement positif sur un secteur $S_{\delta}$.

On rappelle qu'un opérateur $A$ est dit fortement positif si :

-Il existe $\delta$ tel que $0<\delta \leq \pi$ de telle sorte que le secteur $S_{\delta}=\{\lambda \in \mathbb{C} ;|\operatorname{Arg}(\lambda)|$ $>\delta\}$ soit inclus dans l'ensemble résolvant $\rho(A)$ de l'opérateur $A$.

-Il existe une constante $M>0$ tel que $\left\|(A-\lambda I)^{-1}\right\| \leq \frac{M}{1+|\lambda|}$ pour tout $\lambda \in S_{\delta}$.

Remarque 2.5. 1) D’après Balakrishnan [7], un opérateur $A$ fortement positif admet une puissance fractionnaire $A^{\alpha}, 0<\alpha<1$, qui est un opérateur fortement positif.

2) D'aprés le théorème 5.4 Chap I de Krein [16], $-A^{\alpha}$ est un générateur infinitésimal d'un semi-groupe analytique pour $0<\alpha \leq \frac{1}{2}$.

Dans la suite on note par:

$-E\left(A^{\alpha}\right)=\left\{u \in D\left(A^{\alpha}\right) ;\|u\|_{E\left(A^{\alpha}\right)}=\left\|A^{\alpha} u\right\|_{E} ; \alpha>0\right\}$

$-L_{2}([0,1], E)$ est l'espace de Hilbert des fonctions à valeurs dans $E$ de carré intégrable et de norme $\|u\|_{L_{2}([0,1], E)}=\sqrt{\int_{0}^{1}\|u(x)\|_{E}^{2} d x}$.

$-W_{2}^{2}\left([0,1], E\left(A^{m}\right), E\right)=\left\{u ; A^{m} u\right.$ et $\left.u^{\prime \prime} \in L_{2}([0,1], E) ; m \in \mathbb{N}\right\}$ de norme :

$$
\|u\|_{W_{2}^{2}\left([0,1], E\left(A^{m}\right), E\right)}=\left\|A^{m} u\right\|_{L_{2}([0,1], E)}+\left\|u^{\prime \prime}\right\|_{L_{2}([0,1], E)}
$$

$-S i-A$ engendre un semi groupe analytique $e^{-t A}$ pour tout $t>0$, on définit l'espace d'interpolation:

$$
E_{\alpha}=\left[E, E\left(A^{m}\right)\right]_{\alpha}=\left\{u ; \int_{0}^{\infty} t^{2 m(1-\alpha)-1}\left\|A^{m} e^{-t A} u\right\|^{2} d t<\infty\right\} \text { avec } m \in \mathbb{N}
$$


Dans ce travail, on établit un résultat de complétude dans $L_{2}([0,1], E) \mathrm{du}$ système des vecteurs propres généralisés de l'opérateur $T u(x)=-u^{\prime \prime}(x)+A u(x)$ de domaine:

$$
\begin{aligned}
& D(T)=\left\{u \in W_{2}^{2}([0,1], E(A), E) ;\right. B_{1}(u)=u^{\prime}(0)-u^{\prime}(1)+u(1)=0 \text { et } \\
&\left.B_{2}(u)=u(0)+u(1)=0\right\}
\end{aligned}
$$

Théorème 2.6. Pour $u \in L_{2}([0,1], E)$, on pose $B_{1}(u)=u^{\prime}(0)-u^{\prime}(1)+u(1)$ et $B_{2}(u)=u(0)+u(1)$. Soit $T$ un opérateur agissant dans $L_{2}([0,1], E)$ défini par $T u=-u^{\prime \prime}(x)+A u(x)$ de domaine $D(T)=\left\{u \in W_{2}^{2}([0,1], E(A), E) ; B_{1}(u)=\right.$ 0 et $\left.B_{2}(u)=0\right\}$ On suppose:

1) Il existe $p>0$ et $\lambda_{0} \in \rho(A) ;\left(A-\lambda_{0} I\right)^{-1} \in \ell_{p}^{(0)}$

2) Il existe $\delta$ tel que $0<\delta<\frac{\pi}{2(p+2)}$ de sorte que $A$ soit fortement positif sur le secteur $S_{\delta}=\{\lambda \in \mathbb{C} ;|\operatorname{Arg}(\lambda)|>\delta\}$

Alors le système des vecteurs propres généralisés de $T$ est dense dans $L_{2}([0,1], E)$.

La démonstration de ce théorème repose sur les lemmes 2.7 et 2.8 démontrés ci-après.

Lemme 2.7. On suppose que $A$ est fortement positif de secteur $S_{\delta}$. Alors il existe $M>0$ tel que $\left\|(T-\lambda I)^{-1}\right\| \leq \frac{M}{\sqrt{|\lambda|}}$ pour $|\lambda|$ assez grand dans le
secteur $S_{\delta}$.

Démonstration. Soit $f \in L_{2}([0,1], E)$, on considère l'équation :

$$
\left\{\begin{array}{l}
-u^{\prime \prime}(x)+(A-\lambda I) u(x)=f(x), \quad 0 \leq x \leq 1 \\
u \in D(T)
\end{array}\right.
$$

En utilisant la méthode donnée par B. A. Aliev et I. V. Aliev dans [6], on déduit que la solution $u(x)$ de (1) s'écrit sous la forme $u(x)=u_{1}(x)+u_{2}(x)$ où :

$-u_{1}(x)$ est la restriction à l'intervalle $[0,1]$ de la solution de l'équation:

$$
\left\{\begin{array}{l}
-v^{\prime \prime}(x)+(A-\lambda I) v(x)=g(x) \text { pour }-\infty<x<+\infty \\
g(x)=f(x) \text { pour } x \in[0,1] \\
g(x)=0 \text { pour } x \notin[0,1]
\end{array}\right.
$$

$-u_{2}(x)$ est la solution de l'équation:

$$
\left\{\begin{array}{l}
-u_{2}^{\prime \prime}(x)+(A-\lambda I) u_{2}(x)=O, \quad 0 \leq x \leq 1 \\
u_{2}^{\prime}(0)-u_{2}^{\prime}(1)+u_{2}(1)=-B_{1}\left(u_{1}\right) \\
u_{2}(0)+u_{2}(1)=-B_{2}\left(u_{1}\right)
\end{array}\right.
$$


En appliquant le théorème de Mikhlin-Schwartz [5], on déduit que pour tout $\lambda \in S_{\delta}$, il existe une constante $C>0$ indépendante de $\lambda$ telle que:

$$
\left\|u_{1}\right\|_{W_{2}^{2}([0,1], E(A), E)} \leq C\|f\|_{L_{2}([0,1], E)}
$$

On a en particulier:

$$
\left\|(A-\lambda I) u_{1}\right\|_{L_{2}([0,1], E)} \leq C\|f\|_{L_{2}([0,1], E)}
$$

D'où :

$$
\begin{aligned}
\left\|u_{1}\right\|_{L_{2}([0,1], E)} & =\left\|(A-\lambda I)^{-1}(A-\lambda I) u_{1}\right\|_{L_{2}([0,1], E)} \\
& \leq \frac{C}{1+|\lambda|}\left\|(A-\lambda I) u_{1}\right\|_{L_{2}([0,1], E)}
\end{aligned}
$$

et par conséquent:

$$
\left\|u_{1}\right\|_{L_{2}([0,1], E)}=\frac{C}{1+|\lambda|}\|f\|_{L_{2}([0,1], E)}
$$

Pour l'équation (3), on applique le lemme suivant donné par B. A. Aliev et I. V. Aliev dans [6].

Lemme 2.8 (B. A. Aliev et I. V. Aliev [6]). Pour que la solution générale $u_{2}(x)$ de l'équation $-u_{2}^{\prime \prime}(x)+(A-\lambda I) u_{2}(x)=0$ soit dans $W_{2}^{2}([0,1], E(A), E)$ il faut et il suffit qu'elle soit de la forme:

$$
u_{2}(x)=e^{-x A_{\lambda}^{1 / 2}} g_{1}+e^{-(1-x) A_{\lambda}^{1 / 2}} g_{2} \text { où } A_{\lambda}=A-\lambda I \text { et } g_{1}, g_{2} \in[E, E(A)]_{3 / 4} \text {. }
$$

Pour que $u_{2}(x)$ soit solution de (3) il faut qu'elle vérifie les conditions aux limites :

$$
\left\{\begin{array}{l}
{\left[-A_{\lambda}^{1 / 2}+\left(I+A_{\lambda}^{1 / 2}\right) e^{-A_{\lambda}^{1 / 2}}\right] g_{1}+\left(I-A_{\lambda}^{1 / 2}+A_{\lambda}^{1 / 2} e^{-A_{\lambda}^{1 / 2}}\right) g_{2}=-B_{1}\left(u_{1}\right)} \\
\left(I+e^{-A_{\lambda}^{1 / 2}}\right) g_{1}+\left(I+e^{-A_{\lambda}^{1 / 2}}\right) g_{2}=-B_{2}\left(u_{1}\right)
\end{array}\right.
$$

Sous forme analogue à celle donnée dans [5] ce système peut s'écrire:

$$
K_{1}(\lambda)\left(\begin{array}{l}
g_{1} \\
g_{2}
\end{array}\right)+K_{2}(\lambda)\left(\begin{array}{l}
g_{1} \\
g_{2}
\end{array}\right)=\left(\begin{array}{l}
-B_{1}\left(u_{1}\right) \\
-B_{2}\left(u_{1}\right)
\end{array}\right)
$$

où :

$$
K_{1}(\lambda)=\left(\begin{array}{cc}
-A_{\lambda}^{1 / 2} & I-A_{\lambda}^{1 / 2} \\
I & I
\end{array}\right)
$$

et

$$
K_{2}(\lambda)=\left(\begin{array}{cc}
\left(I+A_{\lambda}^{1 / 2}\right) e^{-A_{\lambda}^{1 / 2}} & A_{\lambda}^{1 / 2} e^{-A_{\lambda}^{1 / 2}} \\
e^{-A_{\lambda}^{1 / 2}} & e^{-A_{\lambda}^{1 / 2}}
\end{array}\right)
$$


$K_{1}(\lambda)$ est un opérateur inversible mais d'inverse non borné.

Le vecteur $\left(\begin{array}{l}g_{1} \\ g_{2}\end{array}\right)$ est donné formellement par:

$$
\left(\begin{array}{l}
g_{1} \\
g_{2}
\end{array}\right)=\left[I+K_{1}^{-1}(\lambda) K_{2}(\lambda)\right]^{-1} K_{1}^{-1}(\lambda)\left(\begin{array}{c}
-B_{1}\left(u_{1}\right) \\
-B_{2}\left(u_{1}\right)
\end{array}\right)
$$

où :

$$
K_{1}^{-1}(\lambda) K_{2}(\lambda)=\left(\begin{array}{cc}
2 A_{\lambda}^{1 / 2} e^{-A_{\lambda}^{1 / 2}} & \left(I-2 A_{\lambda}^{1 / 2}\right) e^{-A_{\lambda}^{1 / 2}} \\
\left(I+2 A_{\lambda}^{1 / 2}\right) e^{-A_{\lambda}^{1 / 2}} & 2 A_{\lambda}^{1 / 2} e^{-A_{\lambda}^{1 / 2}}
\end{array}\right)
$$

et

$$
\begin{aligned}
K_{1}^{-1}(\lambda)\left(\begin{array}{c}
-B_{1}\left(u_{1}\right) \\
-B_{2}\left(u_{1}\right)
\end{array}\right) & =\left(\begin{array}{c}
B_{1}\left(u_{1}\right)-B_{2}\left(u_{1}\right)+A_{\lambda}^{1 / 2} B_{2}\left(u_{1}\right) \\
-B_{1}\left(u_{1}\right)-A_{\lambda}^{1 / 2} B_{2}\left(u_{1}\right)
\end{array}\right) \\
& =\left(\begin{array}{c}
u_{1}^{\prime}(0)-u_{1}^{\prime}(1)-u_{1}(0)+A_{\lambda}^{1 / 2} u_{1}(0)+A_{\lambda}^{1 / 2} u_{1}(1) \\
-u_{1}^{\prime}(0)+u_{1}^{\prime}(1)-u_{1}(1)-A_{\lambda}^{1 / 2} u_{1}(0)-A_{\lambda}^{1 / 2} u_{1}(1)
\end{array}\right)
\end{aligned}
$$

Remarque 2.9. -Comme $u_{1} \in W_{2}^{2}([0,1], E(A), E)$, on déduit par interpolation que $u_{1}^{\prime}(0), u_{1}^{\prime}(1), A_{\lambda}^{1 / 2} u_{1}(0), A_{\lambda}^{1 / 2} u_{1}(1)$ appartiennent á $[E, E(A)]_{1 / 4}$ et que $u_{1}(0), u_{1}(1)$ appartiennent á $[E, E(A)]_{3 / 4}$.

-La norme de chacun de ces termes dans $[E, E(A)]_{1 / 4}$ est majorée par celle de $u_{1}$ dans $W_{2}^{2}([0,1], E(A), E)$.

On va donc chercher à majorer la norme de $K_{1}^{-1}(\lambda) K_{2}(\lambda)\left(\begin{array}{l}g_{1} \\ g_{2}\end{array}\right)$ dans $[E, E(A)]_{1 / 4}$. Pour cela il faudra majorer, pour $i=1,2$, les éléments de la forme:

$$
\left\|A_{\lambda}^{1 / 2} e^{-A_{\lambda}^{1 / 2}} g_{i}\right\|_{[E, E(A)]_{1 / 4}} .
$$

De la définition de $[E, E(A)]_{1 / 4}$, on déduit que:

Or

$$
\left\|A_{\lambda}^{1 / 2} e^{-A_{\lambda}^{1 / 2}} g_{i}\right\|_{[E, E(A)]_{1 / 4}} \leq C\left\|g_{i}\right\|_{[E, E(A)]_{3 / 4}}
$$

$$
\left\|g_{i}\right\|_{[E, E(A)]_{3 / 4}}=\left\|A_{\lambda}^{-1 / 2} A_{\lambda}^{1 / 2} g_{i}\right\|_{[E, E(A)]_{3 / 4}}
$$

$$
\leq \frac{C}{\sqrt{1+|\lambda|}}\left\|A_{\lambda}^{1 / 2} g_{i}\right\|_{[E, E(A)]_{3 / 4}}
$$

Comme $\left\|A_{\lambda}^{1 / 2} g_{i}\right\|_{[E, E(A)]_{3 / 4}}=\left\|g_{i}\right\|_{[E, E(A)]_{1 / 4}}$, on obtient la majoration:

$$
\left\|K_{1}^{-1}(\lambda) K_{2}(\lambda)\left(\begin{array}{c}
g_{1} \\
g_{2}
\end{array}\right)\right\|_{[E, E(A)]_{1 / 4}} \leq \frac{C}{\sqrt{1+|\lambda|}}\left\|\left(\begin{array}{c}
g_{1} \\
g_{2}
\end{array}\right)\right\|_{[E, E(A)]_{1 / 4}}
$$


Pour $|\lambda|$ assez grand, la série $\left[I+K_{1}^{-1}(\lambda) K_{2}(\lambda)\right]^{-1}$ est donc convergente et bornée indépendamment de $\lambda$.

D'autre part, d'après la remarque précédente on sait que:

$$
\left\|K_{1}^{-1}(\lambda)\left(\begin{array}{l}
-B_{1}\left(u_{1}\right) \\
-B_{2}\left(u_{1}\right)
\end{array}\right)\right\|_{[E, E(A)]_{1 / 4}} \leq C\left\|u_{1}\right\|_{W_{2}^{2}([0,1], E(A), E)} \leq C\|f\|_{L_{2}([0,1], E)}
$$

où $C$ ne dépend pas de $\lambda$. Il en résulte que:

$$
\left\|\left(\begin{array}{l}
g_{1} \\
g_{2}
\end{array}\right)\right\|_{[E, E(A)]_{1 / 4}} \leq C\|f\|_{L_{2}([0,1], E)}
$$

Pour achever la démonstration du lemme 2.7, il nous reste à majorer la norme de $u_{2}(x)$ dans $L_{2}([0,1], E)$.

$$
\begin{aligned}
\left\|u_{2}(x)\right\|_{L_{2}([0,1], E)} & \left\|A_{\lambda}^{-1 / 2} A_{\lambda}^{1 / 2} u_{2}(x)\right\|_{L_{2}([0,1], E)} \\
& \leq \frac{C}{\sqrt{|\lambda|}}\left\|A_{\lambda}^{1 / 2} u_{2}(x)\right\|_{L_{2}([0,1], E)} \\
& \leq \frac{C}{\sqrt{|\lambda|}}\left\|A_{\lambda}^{1 / 2} e^{-x A_{\lambda}^{1 / 2}} g_{1}+A_{\lambda}^{1 / 2} e^{-(1-x) A_{\lambda}^{1 / 2}} g_{2}\right\|_{L_{2}([0,1], E)} \\
\leq & \frac{C}{\sqrt{|\lambda|}}\left[\left\|A_{\lambda}^{1 / 2} e^{-x A_{\lambda}^{1 / 2}} g_{1}\right\|_{L_{2}([0,1], E)}+\left\|A_{\lambda}^{1 / 2} e^{-(1-x) A_{\lambda}^{1 / 2}} g_{2}\right\|_{L_{2}([0,1], E)}\right]
\end{aligned}
$$

Or :

$$
\left\|A_{\lambda}^{1 / 2} e^{-x A_{\lambda}^{1 / 2}} g_{1}\right\|_{L_{2}([0,1], E)} \leq\left\|A_{\lambda}^{-1 / 2} g_{1}\right\|_{[E, E(A)]_{3 / 4}} \leq\left\|g_{1}\right\|_{[E, E(A)]_{1 / 4}}
$$

et

$$
\left\|A_{\lambda}^{1 / 2} e^{-x A_{\lambda}^{1 / 2}} g_{2}\right\|_{L_{2}([0,1], E)} \leq\left\|A_{\lambda}^{-1 / 2} g_{2}\right\|_{[E, E(A)]_{3 / 4}} \leq\left\|g_{2}\right\|_{[E, E(A)]_{14}}
$$

D'où l'on déduit que:

$$
\left\|u_{2}(x)\right\|_{L_{2}([0,1], E)} \leq \frac{C}{\sqrt{|\lambda|}}\|f\|_{L_{2}([0,1], E)}
$$

Comme $\left\|u_{1}(x)\right\|_{L_{2}([0,1], E)}=\frac{C}{1+|\lambda|}\|f\|_{L_{2}([0,1], E)}$, on obtient :

$$
\|u(x)\|_{L_{2}([0,1], E)} \leq \frac{C}{\sqrt{|\lambda|}}\|f\|_{L_{2}([0,1], E)} .
$$

C'est-à-dire :

$$
\left\|(T-\lambda I)^{-1}\right\|_{L_{2}([0,1], E)} \leq \frac{C}{\sqrt{|\lambda|}}
$$


Lemme 2.10. On suppose qu'il existe $p>0$ et $\lambda_{0} \in \rho(A) ;\left(A-\lambda_{0} I\right)^{-1} \in \ell_{p}^{(0)}$. Alors $(T-\lambda I)^{-1} \in \ell_{n}^{(0)}$ pour $n>p+2$.

Démonstration. On note $S$ l'opérateur $-D^{2}$ auto adjoint, défini positif sur $L_{2}[0,1]$, de domaine $D(S)=W_{2}^{2}[0,1]$ avec les conditions de Stone. Soit $u_{k}(x)$ les fonctions propres de $S$ associées à $\lambda_{k}$. Les valeurs singulières de $S$ vérifient $1 / s_{k}(S)=O\left(k^{-1 / 2}\right)$ quand $k$ tend vers l'infini. De la même façon, il existe un opérateur $R$ auto-adjoint, défini positif dans $E$, de domaine $D(R)=$ $E(A)$. Soit $v_{m}$ les fonctions propres de $R$ associées aux valeurs propres $\sigma_{m}$. Comme il existe $\lambda_{0} \in \rho(A)$ tel que $\left(A-\lambda_{0} I\right)^{-1} \in \ell_{p}^{(0)}$, on en déduit que les valeurs singulières de $R$ vérifient $1 / \mathrm{s}_{m}(R)=O\left(m^{-1 / p}\right)$ quand $m$ tend vers l'infini, c'est-à-dire il existe $\sigma_{0} \in \rho(R) ;\left(A-\sigma_{0} I\right)^{-1} \in \ell_{p}^{(0)}$.

Comme $\left\{u_{k}(x)\right\}$ est une base de $L_{2}[0,1]$ et $\left\{v_{m}\right\}$ une base de $E$, alors $\left\{u_{k}(x) v_{m}\right\}$ est une base de $L_{2}([0,1], E)$. On définit l'opérateur $B u=\sum_{k=1}^{\infty} \sum_{m=1}^{\infty}\left(1+\lambda_{k}+\right.$
$\left.\sigma_{m}\right) a_{k ; m} u_{k}(x) v_{m}$ où

$$
u=\sum_{k=1}^{\infty} \sum_{m=1}^{\infty} a_{k ; m} u_{k}(x) v_{m}
$$

D'une part, pour $n>p+2$ la résolvante de $B$ appartient à $\ell_{n}^{(0)}$ et d'autre part le domaine de $T$ est inclus dans celui de $B$.

On en déduit que l'opérateur $B(T-\lambda I)^{-1}$ est borné dans $L_{2}([0,1], E)$ et par conséquent que $(T-\lambda I)^{-1} \in \ell_{n}^{(0)}$.

La démonstration du théorème précédent se fait en combinant ces deux lemmes et le théorème 1.10 .

On établit de même un résultat de complétude du système des vecteurs propres généralisés dans $L_{2}([0,1], E)$ de l'opérateur $T u=u^{\prime \prime}-A u$ sous les conditions irrégulières de Birkhof $B_{1}(u)=a_{1} u^{\prime}(0)+b_{1} u^{\prime}(1)+a_{0} u(0)+b_{0} u(1)$ $=0$ et $B_{2}(u)=a_{1} u(0)-b_{1} u(1)=0$. Ces conditions sont une généralisation de celles de Stone et vérifient:

$$
a_{1} d_{1}-b_{1} c_{1}=0, a_{0} d_{0}-b_{0} c_{0} \neq 0 \text { et } a_{1} d_{0}-b_{0} c_{1}=a_{0} d_{1}-b_{1} c_{0}
$$

Corollaire 2.11. Pour $u \in L_{2}([0,1], E)$, on pose $B_{1}(u)=a_{1} u^{\prime}(0)+b_{1} u^{\prime}(1)$ $+a_{0} u(0)+b_{0} u(1)$ et $B_{2}(u)=a_{1} u(0)-b_{1} u(1)$. Soit $T$ un opérateur agissant dans $L_{2}([0,1], E)$ défini par $T u=-u^{\prime \prime}(x)+A u(x)$ de domaine:

$$
D(T)=\left\{u \in W_{2}^{2}([0,1], E(A), E) ; B_{1}(u)=0 \text { et } B_{2}(u)=0\right\}
$$

On suppose:

1) Il existe $p>0$ et $\lambda_{0} \in \rho(A) ;\left(A-\lambda_{0} I\right)^{-1} \in \ell_{p}^{(0)}$.

2) $a_{0} b_{1}+a_{1} b_{0} \neq O$

3) Il existe $\delta$ tel que $0<\delta<\frac{\pi}{2(p+2)}$ de sorte que $A$ soit fortement positif 
sur le secteur $S_{\delta}=\{\lambda \in \mathbb{C} ;|\operatorname{Arg}(\lambda)|>\delta\}$

Alors le système des vecteurs propres généralisés de $T$ est dense dans $L_{2}([0,1], E)$.

Remarque 2.12. 1) La généralisation du théorème précédent dans $L_{p}([0,1], E)$ se fait sans aucune difficulté.

2) Si on suppose qu'il existe $n>0$ et $\lambda_{0} \in \rho(A) ;\left(A-\lambda_{0} I\right)^{-1} \in \ell_{n}^{(0)}$, Alors le système des vecteurs propres généralisés de l'opérateur $\mathrm{Tu}(x)=-u^{\prime \prime}(x)+$ $A u(x)$ de domaine:

$D(T)=\left\{u \in W_{2}^{2}([0,1], E(A), E) ; u^{\prime}(1)+B u(0)+T_{1} u=0\right.$ et $\left.u(0)+T_{2} u=0\right\}$ où les opérateurs $A, B, T_{1}$ et $T_{2}$ vérifient les hypothèses données par $B$. A. Aliev et I. $V$. Aliev dans $[6]$, est dense dans $L_{2}([0,1], E)$.

3) Dans [15], une étude est entreprise sur la complétude du système des vecteurs propres généralisés dans $L_{2}([0,1], E)$ de l'opérateur $T u=-u^{\prime \prime}+A u$ sous les conditions de Lang et Locker: $B_{1}(u)=a_{1} u^{\prime}(0)+b_{1} u^{\prime}(1)+a_{0} u(0)+b_{0} u(1)=0$ et $B_{2}(u)=c_{1} u^{\prime}(0)+d_{1} u^{\prime}(1)+c_{0} u(0)+d_{0} u(1)=0$

\section{Références}

[1] Aimar, M-T., Intissar, A. et Paoli, J-M., Densité des vecteurs propres généralisés d'une classe d'opérateurs non auto-adjoints à résolvante compacte, C. R. Acad. Sci. Paris, Série I, 315 (1992), 393-396.

[2] - Densité des vecteurs propres généralisés d'une classe d'opérateurs compacts non auto-adjoints et applications, Comm. Math. Phys., 156 (1993), 169-177.

[3] - Quelques propriétés de régularité de l'opérateur de Gribov, C. R. Acad. Sci. Paris, Série I, 320 (1995), 15-18.

[4] —, Quelques nouvelles propriétés de régularité de l'opérateur de Gribov, Comm. Math. Phys, 172 (1995), 461-466.

[5] Aliev, B. A. and Yakubov, S. YA., A boundary-value problem with an operator in the boundary conditions, for a second order elliptic differential-operator equation, Siberian. Math. J., 26, $\mathrm{N}^{\circ} 4$ (1985), 618-628.

[6] Aliev, B. A. and Aliev, I. V., Coercive solvability of boundary-value problems for elliptic operator-differential equations with an operator in boundary conditions, Siberian. Math. J., 31, $\mathrm{N}^{\circ} 4$ (1990), 529-534.

[7] Balakrishnan, A. V., Fractional powers of closed operators and the semigroups generated by them, Pacific. J. Math., 10 (1960), 419-437.

[8] Bargmann, V., On a Hilbert space of analytic functions and an associated integral transform I, Comm. Pure Appl. Math., 14 (1962), 187-214.

[9] Dunford, N. and Schwartz, J. T., Linear operators, II, in Pure Appl. Math., Interscience, New-York, 7 (1963).

[10] Gribov, V. N., A reggeon diagram technique, J.E.T.P. Sov. Phy's, 26 (1968), 414423.

[11] Gohberg, I. C. and Krein, M. G., Introduction to the theory of linear non-self adjoint operators, Providence, A.M.S., 18 (1969), 378.

[12] Intissar, A., Etude spectrale d'une famille d'opérateurs non-symétriques intervenant dans la théorie des champs de reggeons, Comm. Math. Phys., 113 (1987), 263-297.

[13] —, Quelques nouvelles propriétés spectrales de l'hamiltonien de la théorie des 
champs de reggeons, C. R. Acad. Sci. Paris, Série I, 308 (1989), 209-214.

[14] Intissar, A., Théorie spectrale dans l'espace de Bargmann, Cours de D.E.A, Université de Besançon, 1989.

[15] - Preprint.

[16] Krein, S. G., Linear differential equations in Banach space, Providence, A.M.S., 29 (1971), 390.

[17] Lang, P. and Locker, J., Spectral theory of two point differential operators determined by $-D^{2}$, spectral properties. Part I, J. Math. Anal. Appl., 141 (1989), 538-558.

[18] - Denseness of the generalized eigenvectors of an H-S discrete operator, J. Funct. Anal., 82, № 2 (1989), 316-329.

[19] Paoli, J-M., Conditions suffisantes de densité du sous espace engendré par les vecteurs propres généralisés d'un opérateur compact ou à résolvante compacte sur un espace de Hilbert et applications aux opérateurs de Gribov. Thèse, Université de Corté, 1993. 
